\title{
Asymptotic Solutions of the Kinetic Boltzmann Equation and Multicomponent Non-Equilibrium Gas Dynamics
}

\author{
S. A. Serov' ${ }^{1}$, S. S. Serova ${ }^{2}$ \\ ${ }^{1}$ Fundamental Researches Department, Russian Federal Nuclear Centre, All-Russian Scientific Research \\ Institute of Experimental Physics, Sarov, Russia \\ ${ }^{2}$ St. Petersburg State University, St. Petersburg, Russia \\ Email: serov@vniief.ru, serova.s.svetlana@gmail.com
}

Received 16 June 2016; accepted 24 August 2016; published 31 August 2016

\begin{abstract}
In the article correct method for the kinetic Boltzmann equation asymptotic solution is formulated, the Hilbert's and Enskog's methods are discussed. The equations system of multicomponent nonequilibrium gas dynamics is derived, that corresponds to the first order in the approximate (asymptotic) method for solution of the system of kinetic Boltzmann equations.
\end{abstract}

\section{Keywords}

Kinetic Boltzmann Equation, Multicomponent Non-Equilibrium Gas Dynamics

\section{Introduction}

In 1912 Hilbert considered the kinetic Boltzmann equation for one-component gas as an example of integral equation and proposed a "recipe" for its approximate (asymptotic) solution (see [1], Chapter XXII). Hilbert's "recipe" was inconvenient for practical use, because the five arbitrary functional parameters of the first and the following approximations of the velocity distribution function had to be found by solving the differential equations in partial derivatives (equations of gas dynamics of the first and higher orders). Five years later Enskog proposed to use zero conditions, conditions with zero right-hand sides, to determine the five arbitrary functional parameters of the first and following approximations of the velocity distribution function. The imposition of the zero conditions leads, in fact, to using different comparison scales in the asymptotic expansion of the velocity distribution function and in the asymptotic expansion of the particle number density, the mean (mass) velocity and the temperature, that are derived from the asymptotic expansion of the velocity distribution function by integration over velocities with different weighting functions. As a result of paralogism of the method of successive approximations (one has to set variable coefficients of the same terms of the unified comparison scale equal to each other) partial time derivatives vanish in the necessary conditions of solutions existence of integral equations of higher orders (see below) and with them terms of gas-dynamic equations, corresponding to viscosity, heat conduction, ... vanish. Enskog "improved" the situation by the introducing (see, for example, [2], Chapter 7, $\S 1$, Section 5) of the unsubstantiated expansion of partial time derivative: 


$$
\frac{\partial}{\partial t}=\sum_{r=0}^{\infty} \theta^{r} \frac{\partial_{r}}{\partial t}
$$

The approach of Struminskii, who had proposed in 1974 in [3] his approximate (asymptotic) method of solution of the system of kinetic Boltzmann equations for multicomponent gas, differs from the approach of Enskog to asymptotic solution of the Boltzmann equations system for gas mixture in that, how the infinitesimal parameter is introduced in the Boltzmann equations system for gas mixture, i.e. the solution is constructing in another asymptotic limit. In substance, Struminskii's method of solution of kinetic equations system is the same as Enskog's method (Struminskii used the partial time derivative expansion, as Enskog did).

In section 2 below will be proposed the correct method of asymptotic solution of the kinetic Boltzmann equations system for multicomponent gas mixture for the approach, that combines Enskog's and Struminskii's approaches; in particular, it will be shown, how one has to modify Enskog's method: in addition to asymptotic expansion of the velocity distribution function $i$-component particles of gas mixture it is necessary to determine and to use the expansion of the particle number density $n_{i}$ of $i$-component, mean mass velocity $\mathbf{u}$ and temperature $T$ of the gas mixture.

Further, in the Section 3 the system of infinitesimal first order equations of multicomponent non-equilibrium gas dynamics, appearing during the process of the solution of the system of Boltzmann equations by successive approximations method in the Section 2 as necessary condition of the existence of approximate (asymptotic) solution of the integral equations system, is considered in more detail.

This article is condensed version of our article arXiv:1303.6275. Notations, used below, are close to notations in [2]; it is assumed, that all regarded functions are continuous and continuously differentiable so many times as it is necessary, if their derivatives are considered, and all regarded integrals converge.

\section{Correct Method of Solution of the Kinetic Boltzmann Equations System}

The Boltzmann equations system, that describes change of dependent on $t$ and spatial coordinates, prescribed by radius-vector $\mathbf{r}$, the velocity distribution functions $f_{i}\left(t, \mathbf{r}, \mathbf{c}_{i}\right)$ due to collision with particles of other components of mixture of rarefied monatomic gases, where $\mathbf{c}_{i}$ are the velocities of particles of $i$-component of the mixture \{see [2], Chapter 8, Equation (1.1); discussion of the derivation of the Boltzmann equations system and its applicability range see, for example, in [2], Chapters 3 and 18, [4], Chapter 7, § 1; below the central interaction of molecules are considered only, when the force, with which each molecule acts on the other, is directed along the line, connecting the centers of the molecules\}, could be written as:

$$
\begin{aligned}
\frac{\partial f_{i}}{\partial t}+\mathbf{c}_{i} \cdot \frac{\partial f_{i}}{\partial \mathbf{r}}+\frac{\mathbf{X}_{i}}{m_{i}} \cdot \frac{\partial f_{i}}{\partial \mathbf{c}_{i}} & =\sum_{j \in N} \iiint\left(f_{i}^{\prime} f_{j}^{\prime}-f_{i} f_{j}\right) g_{i j} b d b d \varepsilon d \mathbf{c}_{j} \\
& =\sum_{j \in N} \iint\left(f_{i}^{\prime} f_{j}^{\prime}-f_{i} f_{j}\right) k_{i j} d \mathbf{k} d \mathbf{c}_{j} \quad(i \in N) ;
\end{aligned}
$$

in (2) $N$ is a set of indexes, that are numbering components of the mixture; $\mathbf{X}_{i}$ is an external force, which acts on the molecule of the $i$-component; $m_{i}$ is the mass of the molecule of the $i$-component; $g_{i j}$ is the modulus of the relative velocity of colliding particles $\mathbf{g}_{i j}=\mathbf{c}_{i}-\mathbf{c}_{j}$; $b$ is the impact distance, $\varepsilon$ is the azimuth angle, $\mathbf{k}$ is the unit vector, directed to the center of mass of the colliding particles from the point of closest approach — see [2], Chapter 3, Figure 3; the scalar function $k_{i j}\left(\mathbf{g}_{i j}, \mathbf{k}\right)$ is determined by equality

$$
g_{i j} b d b d \varepsilon \stackrel{\text { def }}{=} k_{i j} d \mathbf{k} \text {; }
$$

by prime in (20) and below the velocities and the functions of velocities after the collision are denoted.

Let us introduce following notations:

$$
\begin{gathered}
J_{i}\left(f_{i}, f\right)=\iint\left(f_{i} f-f_{i}^{\prime} f^{\prime}\right) k_{i} d \mathbf{k} d \mathbf{c}, \\
J_{i j}\left(f_{i}, f_{j}\right)=\iint\left(f_{i} f_{j}-f_{i}^{\prime} f_{j}^{\prime}\right) k_{i j} d \mathbf{k} d \mathbf{c}_{j} ;
\end{gathered}
$$

to differ velocities of colliding molecules of the same kind in (22) the one velocity is denoted by $\mathbf{c}_{j}$ and the other is denoted by $\mathbf{c}$ (without any index) and the index of the corresponding velocity distribution function $f$ is omitted. 
In Enskog's approach the differential parts of the Boltzmann Equations (2), that are denoted by $\mathcal{D}_{i} f_{i}$ below, are considered to be small as compared with the right-hand sides of Equations (2) — see [2], Chapter 7, § 1, Section 5-therefore the indicator of infinite smallness $\theta$ is formally introduced in the Boltzmann equations system in the following way:

$$
\theta \mathcal{D}_{i} f_{i}=-\sum_{j} J_{i j}\left(f_{i}, f_{j}\right) \quad(i \in N) .
$$

In Struminskii's approach to the asymptotic solution of the Boltzmann equations system the differential parts of the Boltzmann Equations (2) and the collision integrals of the particles of $i$-component with the particles of the other components are considered to be small as compared with the collision integral of the particles of $i$-component between each other, therefore the indicator of infinitesimality $\theta$ is introduced in the Boltzmann equations system in another way:

$$
\theta \mathcal{D}_{i} f_{i}=-J_{i}\left(f_{i}, f\right)-\theta \sum_{j \neq i} J_{i j}\left(f_{i}, f_{j}\right) \quad(i \in N) .
$$

It is possible to combine Enskog's approach with Struminskii's approach. For this purpose we divide the set of mixture components $N$ into two subsets: the subset of components, that we call formally inner components (we could consider the case, when there are some subsets of inner components, but this case does not fundamentally differ from the one, considered below, the only difference is that the notation become more complicated) and the subset of components, that we call external components. To differ the two groups of mixture components we denote the subset of indexes of inner components $\hat{N}$ as well as the indexes of inner components $\hat{i} \in \hat{N}$ and the subset of indexes of external components $\breve{N}$ as well as the indexes of external components $\breve{i} \in \bar{N}$; the intersection of the sets $\hat{N}$ and $\breve{N}$ is the empty set $-\hat{N} \cap \breve{N}=\varnothing$ and the union of these sets is the set indexes of all mixture components $\hat{N} \cup \breve{N}=N$; if an assertion concerns both kinds of components the special symbols will be omitted. In new notations the Boltzmann equations system can be rewritten as:

$$
\begin{array}{cc}
\theta \mathcal{D}_{\hat{i}} f_{\hat{i}}=-\sum_{\hat{j} \in \hat{N}} J_{\hat{i}}\left(f_{\hat{i}}, f_{\hat{j}}\right)-\theta \sum_{j \in N} J_{\hat{i} j}\left(f_{\hat{i}}, f_{\breve{j}}\right) & (\hat{i} \in \hat{N}), \\
\theta \mathcal{D}_{\grave{i}} f_{\breve{i}}=-J_{\breve{i}}\left(f_{\breve{i}}, f\right)-\theta \sum_{j \neq \grave{i}} J_{\breve{i} j}\left(f_{\tilde{i}}, f_{j}\right) \quad(\breve{i} \in \breve{N}) .
\end{array}
$$

Let us write the asymptotic expansion of the velocity distribution function $f_{i}$ of particles of $i$-component as formal series of successive approximations in powers of $\theta$ :

$$
f_{i}=f_{i}^{(0)}+\theta f_{i}^{(1)}+\theta^{2} f_{i}^{(2)}+\cdots
$$

The differential parts of the Equations (3) are written as:

$$
\begin{aligned}
\mathcal{D}_{i} f_{i} & =\left(\frac{\partial}{\partial t}+\mathbf{c}_{i} \cdot \frac{\partial}{\partial \mathbf{r}}+\frac{\mathbf{X}_{i}}{m_{i}} \cdot \frac{\partial}{\partial \mathbf{c}_{i}}\right)\left(f_{i}^{(0)}+\theta f_{i}^{(1)}+\cdots\right) \\
& =\mathcal{D}_{i}^{(0)}+\theta \mathcal{D}_{i}^{(1)}+\theta^{2} \mathcal{D}_{i}^{(2)}+\cdots,
\end{aligned}
$$

where

$$
\mathcal{D}_{i}^{(r)}=\frac{\partial f_{i}^{(r)}}{\partial t}+\mathbf{c}_{i} \cdot \frac{\partial f_{i}^{(r)}}{\partial \mathbf{r}}+\frac{\mathbf{X}_{i}}{m_{i}} \cdot \frac{\partial f_{i}^{(r)}}{\partial \mathbf{c}_{i}} \quad(r=0,1,2, \ldots),
$$

—cf. with [2], Chapter 7, $\S 1$, Sections 4, 5 and [3]. In (11)-(12) the partial time derivative expansion (1) is not used in contrast to that, how it was made by Enskog and further by Struminskii. As result, described below method for solution of the system of kinetic Boltzmann equations differ fundamentally from Enskog's method and Struminskii's method.

Substituting (10) and (11) in (8) and equating coefficients at the same powers of $\theta$ to each other, we obtain the equations system of the method of successive approximations for finding the velocity distribution functions of inner components particles of gas mixture $f_{\hat{i}}^{(r)}$; taking introduced notations (4), (5) and (12) into account, the system can be rewritten as: 


$$
\begin{gathered}
\sum_{\hat{j} \in \hat{N}} J_{\hat{i} j}\left(f_{\hat{i}}^{(0)}, f_{\hat{j}}^{(0)}\right)=0 \quad(\hat{i} \in \hat{N}), \\
\mathcal{D}_{\hat{i}}^{(r-1)}+\sum_{\hat{j} \in \hat{N}} J_{\hat{i}}\left(f_{\hat{i}}^{(r)}, f_{\hat{j}}^{(0)}\right)+\sum_{\hat{j} \in \hat{N}} \sum_{s=1}^{r-1} J_{\hat{i}}\left(f_{\hat{i}}^{(r-s)}, f_{\hat{j}}^{(s)}\right)+\sum_{\hat{j} \in \hat{N}} J_{\hat{j}}\left(f_{\hat{i}}^{(0)}, f_{\hat{j}}^{(r)}\right) \\
+\sum_{\hat{j} \in \hat{N} s=0}^{r-1} \sum_{\hat{i}}\left(f_{\hat{i}}^{(r-1-s)}, f_{\hat{j}}^{(s)}\right)=0 \quad(\hat{i} \in \hat{N}, r=1,2, \ldots) .
\end{gathered}
$$

Similarly substituting (10) and (11) in (9) and equating coefficients at the same powers of $\theta$ to each other, we obtain the equations system of the method of successive approximations for finding the velocity distribution functions of particles of external components of gas mixture $f_{\bar{i}}^{(r)}$ :

$$
\begin{gathered}
J_{\breve{i}}\left(f_{\bar{i}}^{(0)}, f^{(0)}\right)=0 \quad(\breve{i} \in \breve{N}), \\
\mathcal{D}_{\bar{i}}^{(r-1)}+J_{\breve{i}}\left(f_{\bar{i}}^{(r)}, f^{(0)}\right)+\sum_{s=1}^{r-1} J_{\breve{i}}\left(f_{\breve{i}}^{(r-s)}, f^{(s)}\right)+J_{\breve{i}}\left(f_{\breve{i}}^{(0)}, f^{(r)}\right) \\
+\sum_{j \neq i}^{r-1} \sum_{s=0}^{r} J_{\breve{j}}\left(f_{\bar{i}}^{(r-1-s)}, f_{j}^{(s)}\right)=0 \quad(\breve{i} \in \breve{N}, r=1,2, \ldots) .
\end{gathered}
$$

Speaking about an order of approximation below, we assume the order to be equal to the value of index $r$ in (14), (16). According to (5), (13), in zero order approximation we have the following system of integral equations to find the velocity distribution functions of particles of inner components of gas mixture $f_{\hat{i}}^{(0)}$ :

$$
\sum_{\hat{j} \in \hat{N}} J_{\hat{i}}\left(f_{\hat{i}}^{(0)}, f_{\hat{j}}^{(0)}\right)=\sum_{\hat{j} \in \hat{N}} \iint\left(f_{\hat{i}}^{(0)} f_{\hat{j}}^{(0)}-f_{\hat{i}}^{(0)^{\prime}} f_{\hat{j}}^{(0)^{\prime}}\right) k_{\hat{i} j} d \mathbf{k} d \mathbf{c}_{\hat{j}}=0 \quad(\hat{i} \in \hat{N}) .
$$

The general solution of the equations system (17) can be written as a set of the Maxwell functions:

$$
f_{\hat{i}}^{(0)}=\beta_{\hat{i}}^{(1,0)}\left(\frac{m_{\hat{i}}}{2 \pi k \beta^{(3,0)}}\right)^{3 / 2} e^{\frac{m_{\hat{i}}\left(\mathbf{c}_{\hat{i}}-\mathbf{b}^{(2,0)}\right)^{2}}{2 k \beta^{(3,0)}}} \quad(\hat{i} \in \hat{N}),
$$

where $k$ is the Boltzmann constant.

Particle number density $n_{i}$ of the $i$-component, mean mass velocity $\mathbf{u}$ and temperature $T$ of inner components of mixture are introduced by definitions:

$$
\begin{gathered}
n_{i} \stackrel{\operatorname{def}}{=} \int f_{i} d \mathbf{c}_{i}, \\
\mathbf{u} \sum_{\hat{i} \in \hat{N}} n_{\hat{i}} m_{\hat{i}} \stackrel{\text { def }}{=} \sum_{\hat{i} \in \hat{N}} \int m_{\hat{i}} \mathbf{c}_{\hat{i}} f_{\hat{i}} d \mathbf{c}_{\hat{i}}, \\
\frac{3}{2} k T \sum_{\hat{i} \in \hat{N}} n_{\hat{i}} \stackrel{\text { def }}{=} \sum_{\hat{i} \in \hat{N}} \int_{\frac{1}{2}} \frac{1}{2} m_{\hat{i}}\left(\mathbf{c}_{\hat{i}}-\mathbf{u}\right)^{2} f_{\hat{i}} d \mathbf{c}_{\hat{i}},
\end{gathered}
$$

in (21) $k$ is the Boltzmann constant. From (19)-(21) the equality is obtained:

$$
\frac{3}{2} k T \sum_{\hat{i} \in \hat{N}} n_{\hat{i}}+\frac{1}{2} u^{2} \sum_{\hat{i} \in \hat{N}} n_{\hat{i}} m_{\hat{i}}=\sum_{\hat{i} \in \hat{N}} \int_{2}^{1} m_{\hat{i}} c_{\hat{i}}^{2} f_{\hat{i}} d \mathbf{c}_{\hat{i}},
$$

that is convenient to use below instead of definition (21).

According to definitions (19), (20), (21), in addition to the asymptotic expansion (10) it is necessary to determine asymptotic expansions for particle number density $n_{i}$ of the $i$-component

$$
n_{i}=n_{i}^{(0)}+\theta n_{i}^{(1)}+\theta^{2} n_{i}^{(2)}+\cdots,
$$

mean mass velocity $\mathbf{u}$ 


$$
\mathbf{u}=\mathbf{u}^{(0)}+\theta \mathbf{u}^{(1)}+\theta^{2} \mathbf{u}^{(2)}+\cdots
$$

and temperature $T$ of inner components of mixture

$$
T=T^{(0)}+\theta T^{(1)}+\theta^{2} T^{(2)}+\cdots .
$$

Substituting (10) and (23)-(25) in (19), (20), (22) and equating terms of the same infinitesimal order we obtain Card $(\hat{N})+4$ scalar relations, that connect asymptotic expansions (10) and (23)-(25):

$$
\begin{aligned}
\int f_{\hat{i}}^{(r)} d \mathbf{c}_{\hat{i}}=n_{\hat{i}}^{(r)} \quad(\hat{i} \in \hat{N}), & \\
\sum_{\hat{i} \in \hat{N}} m_{i} \mathbf{c}_{\hat{i}} f_{\hat{i}}^{(r)} d \mathbf{c}_{\hat{i}}= & \sum_{\hat{i} \in \hat{N}} m_{\hat{i}}\left(n_{\hat{i}} \mathbf{u}\right)^{(r)}=\sum_{\hat{i} \in \hat{N}} m_{\hat{i}} \sum_{s=0}^{r} n_{\hat{i}}^{(r-s)} \mathbf{u}^{(s)}=\sum_{s=0}^{r} \hat{\rho}^{(r-s)} \mathbf{u}^{(s)}, \\
\sum_{\hat{i} \in \hat{N}} \int_{\frac{1}{2}} \frac{1}{2} m_{\hat{i}} c_{\hat{i}}^{2} f_{\hat{i}}^{(r)} d \mathbf{c}_{\hat{i}} & =\frac{3}{2} k \sum_{\hat{i} \in \hat{N}}\left(n_{\hat{i}} T\right)^{(r)}+\frac{1}{2} \sum_{\hat{i} \in \hat{N}} m_{\hat{i}}\left(n_{\hat{i}} u^{2}\right)^{(r)} \\
& =\frac{3}{2} k \sum_{\hat{i} \in \hat{N} s=0}^{r} \sum_{\hat{i}}^{(r-s)} T^{(s)}+\frac{1}{2} \sum_{\hat{i} \in \hat{N}} m_{\hat{i}} \sum_{s=0}^{r} \sum_{q=0}^{s} n_{\hat{i}}^{(r-s)} \mathbf{u}^{(s-q)} \cdot \mathbf{u}^{(q)} \\
& =\frac{3}{2} k \sum_{s=0}^{r} \hat{n}^{(r-s)} T^{(s)}+\frac{1}{2} \sum_{s=0}^{r} \sum_{s=0}^{s} \hat{\rho}^{(r-s)} \mathbf{u}^{(s-q)} \cdot \mathbf{u}^{(q)} .
\end{aligned}
$$

In (27), (28) the notations are introduced

$$
\begin{aligned}
\hat{\rho}^{(r-s)} & =\sum_{\hat{i} \in \hat{N}} m_{i} n_{\hat{i}}^{(r-s)}, \\
\hat{n}^{(r-s)} & =\sum_{\hat{i} \in \hat{N}} n_{\hat{i}}^{(r-s)} .
\end{aligned}
$$

In particular, for $r=0$ from (26)-(28) we obtain expressions for arbitrary functions $\beta_{\hat{i}}^{(1,0)}(\mathbf{r}, t), \mathbf{b}^{(2,0)}(\mathbf{r}, t)$ and $\beta^{(3,0)}(\mathbf{r}, t)$ in (18) through the zero order approximations to local values of the $\hat{i}$-component number density, the mean mass velocity and the temperature of inner components of the mixture:

$$
\begin{aligned}
& \beta_{\hat{i}}^{(1,0)}(\mathbf{r}, t)=n_{\hat{i}}^{(0)}(\mathbf{r}, t), \\
& \mathbf{b}^{(2,0)}(\mathbf{r}, t)=\mathbf{u}^{(0)}(\mathbf{r}, t), \\
& \beta^{(3,0)}(\mathbf{r}, t)=T^{(0)}(\mathbf{r}, t) .
\end{aligned}
$$

According to (4), (15), zero order integral equations, from which the velocity distribution functions $f_{\tilde{i}}^{(0)}$ of particles of outer components of the mixture are found:

$$
J_{\breve{i}}\left(f_{\bar{i}}^{(0)}, f^{(0)}\right)=\iint\left(f_{\bar{i}}^{(0)} f^{(0)}-f_{\bar{i}}^{(0)^{\prime}} f^{(0)^{\prime}}\right) k_{i} d \mathbf{k} d \mathbf{c}=0 \quad(\breve{i} \in \breve{N}),
$$

-are simpler than Equations (17) and differ actually from (17) only by lack of summation over components. Therefore, similarly (18), the general solution of the equations system (34) can be written as a set of the Maxwell functions:

$$
f_{\bar{i}}^{(0)}=\beta_{\bar{i}}^{(1,0)}\left(\frac{m_{\bar{i}}}{2 \pi k \beta_{\bar{i}}^{(3,0)}}\right)^{3 / 2} e^{-\frac{m_{\bar{i}}\left(\mathbf{c}_{\bar{i}}-\mathbf{b}_{\bar{i}}^{(2,0)}\right)^{2}}{2 k \beta_{i}^{(3,0)}}} \quad(\breve{i} \in \breve{N}),
$$

where $\beta_{i}^{(1,0)}$ and $\beta_{\bar{i}}^{(3,0)}$ are some, independent of $\mathbf{c}_{\tilde{i}}$, scalar functions of spatial coordinates, defined by the radius vector $\mathbf{r}$, and time $t$, and $\mathbf{b}_{i}^{(2,0)}$ is a vector function of $\mathbf{r}$ and $t$.

Let's add to the definition of the number density of particles of $i$-component definitions of mean velocity $\mathbf{u}_{\bar{i}}$ and temperature $T_{\tilde{i}}$ of outer component of mixture: 


$$
\begin{gathered}
\mathbf{u}_{\breve{i}} n_{\breve{i}} m_{\breve{i}}^{\stackrel{\operatorname{def}}{=}} \int m_{\bar{i}} \mathbf{c}_{\breve{i}} f_{\bar{i}} d \mathbf{c}_{\breve{i}}, \\
\frac{3}{2} k T_{\breve{i}} n_{\breve{i}}^{\stackrel{\operatorname{def}}{=}} \int \frac{1}{2} m_{\breve{i}}\left(\mathbf{c}_{\breve{i}}-\mathbf{u}_{\breve{i}}\right)^{2} f_{\breve{i}} d \mathbf{c}_{\breve{i}} ;
\end{gathered}
$$

from (19), (36), (37) the equality is obtained:

$$
\frac{3}{2} k T_{\bar{i}} n_{\bar{i}}+\frac{1}{2} u_{\bar{i}}^{2} n_{\bar{i}} m_{\bar{i}}=\int \frac{1}{2} m_{\bar{i}} c_{\bar{i}}^{2} f_{\bar{i}} d \mathbf{c}_{\bar{i}},
$$

that is convenient to use below instead of definition (37).

Let's enter similar (24)-(25) asymptotic expansions of outer $\breve{i}$-component mean velocity $\mathbf{u}_{\breve{i}}$

$$
\mathbf{u}_{\bar{i}}=\mathbf{u}_{\bar{i}}^{(0)}+\theta \mathbf{u}_{\bar{i}}^{(1)}+\theta^{2} \mathbf{u}_{\bar{i}}^{(2)}+\cdots
$$

and outer $\breve{i}$-component temperature $T_{\breve{i}}$

$$
T_{\breve{i}}=T_{\grave{i}}^{(0)}+\theta T_{\bar{i}}^{(1)}+\theta^{2} T_{\bar{i}}^{(2)}+\cdots
$$

Substituting (10), (23), (39), (40) in (19), (36), (38) and equating terms of the same infinitesimal order we obtain for each $\breve{i} 5$ (scalar) relations, that connect asymptotic expansions (10), (23), (39), (40):

$$
\begin{aligned}
& \int f_{\breve{i}}^{(r)} d \mathbf{c}_{\breve{i}}=n_{\breve{i}}^{(r)}, \\
& \int m_{\bar{i}} \mathbf{c}_{\bar{i}} f_{\bar{i}}^{(r)} d \mathbf{c}_{\check{i}}=m_{\bar{i}}\left(n_{\bar{i}} \mathbf{u}_{\bar{i}}\right)^{(r)}=m_{\bar{i}} \sum_{s=0}^{r} n_{\bar{i}}^{(r-s)} \mathbf{u}_{\bar{i}}^{(s)}=\sum_{s=0}^{r} \rho_{\bar{i}}^{(r-s)} \mathbf{u}_{\bar{i}}^{(s)} \text {, } \\
& \int \frac{1}{2} m_{\breve{i}} c_{\breve{i}}^{2} f_{\grave{i}}^{(r)} d \mathbf{c}_{\breve{i}}=\frac{3}{2} k\left(n_{\breve{i}} T_{\breve{i}}\right)^{(r)}+\frac{1}{2} m_{\breve{i}}\left(n_{\bar{i}} u_{\grave{i}}^{2}\right)^{(r)} \\
& =\frac{3}{2} k \sum_{s=0}^{r} n_{\bar{i}}^{(r-s)} T_{\bar{i}}^{(s)}+\frac{1}{2} m_{\bar{i}} \sum_{s=0}^{r} \sum_{q=0}^{s} n_{\bar{i}}^{(r-s)} \mathbf{u}_{\bar{i}}^{(s-q)} \cdot \mathbf{u}_{\bar{i}}^{(q)} \\
& =\frac{3}{2} k \sum_{s=0}^{r} n_{\grave{i}}^{(r-s)} T_{\grave{i}}^{(s)}+\frac{1}{2} \sum_{s=0}^{r} \sum_{q=0}^{s} \rho_{\grave{i}}^{(r-s)} \mathbf{u}_{\bar{i}}^{(s-q)} \cdot \mathbf{u}_{\grave{i}}^{(q)},
\end{aligned}
$$

cf. with (26)-(28). In (42), (43) the notation is used

$$
\rho_{\bar{i}}^{(r-s)}=m_{\bar{i}} n_{\bar{i}}^{(r-s)} .
$$

For $r=0$ from (41)-(43) we obtain expressions for arbitrary functions $\beta_{\dot{i}}^{(1,0)}(\mathbf{r}, t), \mathbf{b}_{\dot{i}}^{(2,0)}(\mathbf{r}, t)$ and $\beta_{i}^{(3,0)}(\mathbf{r}, t)$ in (35) through the zero order approximations to local values of the number density, the mean velocity and the temperature of outer $\breve{i}$-component of the mixture:

$$
\begin{aligned}
& \beta_{\dot{i}}^{(1,0)}(\mathbf{r}, t)=n_{\bar{i}}^{(0)}(\mathbf{r}, t), \\
& \mathbf{b}_{\bar{i}}^{(2,0)}(\mathbf{r}, t)=\mathbf{u}_{\bar{i}}^{(0)}(\mathbf{r}, t), \\
& \beta_{\bar{i}}^{(3,0)}(\mathbf{r}, t)=T_{\breve{i}}^{(0)}(\mathbf{r}, t) .
\end{aligned}
$$

For $r \geq 1$ the velocity distribution functions of inner components of gas mixture $f_{\hat{i}}^{(r)}$ are found from the integral equations system (14), which, taking (5) and equality

$$
f_{\hat{i}}^{(0)^{\prime}} f_{\hat{j}}^{(0)^{\prime}} \equiv f_{\hat{i}}^{(0)} f_{\hat{j}}^{(0)}
$$

into account, can be rewritten in the form

$$
\begin{aligned}
& \mathcal{D}_{\hat{i}}^{(r-1)}+\sum_{\hat{j} \in \hat{N}} \sum_{s=1}^{r-1} J_{\hat{i} j}\left(f_{\hat{i}}^{(r-s)}, f_{\hat{j}}^{(s)}\right)+\sum_{\hat{j} \in \bar{N} s=0}^{r-1} \sum_{i \hat{i j}}\left(f_{\hat{i}}^{(r-1-s)}, f_{\hat{j}}^{(s)}\right)=-\sum_{\hat{j} \in \hat{N}} J_{\hat{i} j}\left(f_{\hat{i}}^{(0)} \chi_{\hat{i}}^{(r)}, f_{\hat{j}}^{(0)}\right)-\sum_{\hat{j} \in \hat{N}} J_{\hat{i} j}\left(f_{\hat{i}}^{(0)}, f_{\hat{j}}^{(0)} \chi_{\hat{j}}^{(r)}\right) \\
& =-\sum_{\hat{j} \in \hat{N}} \iint_{\hat{i}}^{(0)} f_{\hat{j}}^{(0)}\left(\chi_{\hat{i}}^{(r)}+\chi_{\hat{j}}^{(r)}-\chi_{\hat{i}}^{(r)^{\prime}}-\chi_{\hat{j}}^{(r)^{\prime}}\right) k_{\hat{i} j} d \mathbf{k} d \mathbf{c}_{\hat{j}} \quad(\hat{i} \in \hat{N}),
\end{aligned}
$$


in (49) functions $f_{\hat{i}}^{(r)}$ are written as $f_{\hat{i}}^{(r)}=f_{\hat{i}}^{(0)} \chi_{\hat{i}}^{(r)}$, where $\chi_{\hat{i}}^{(r)}$ are new unknown functions.

The left-hand sides of Equations (49) involves functions, that are known from the previous step of the successive approximations method. Unknown functions $\chi_{\hat{i}}^{(r)}$ enter, linearly, only into the right-hand sides of Equations (49). Therefore the general solution of the system of Equations (14) is a family of functions of a form $\left\{f_{\hat{i}}^{(r)}=\Xi_{\hat{i}}^{(r)}+\xi_{\hat{i}}^{(r)}\right\}_{\hat{i} \in \hat{N}}$, where $\left\{\Xi_{\hat{i}}^{(r)}=f_{\hat{i}}^{(0)} \Phi_{\hat{i}}^{(r)}\right\}_{\hat{i} \in \hat{N}},\left\{\xi_{\hat{i}}^{(r)}=f_{\hat{i}}^{(0)} \phi_{\hat{i}}^{(r)}\right\}_{\hat{i} \in \hat{N}}$, a family of functions $\left\{\Phi_{\hat{i}}^{(r)}\right\}_{\hat{i} \in \hat{N}}$ is a particular solution of the system of inhomogeneous Equations (49) and a family of functions $\left\{\phi_{\hat{i}}^{(r)}\right\}_{\hat{i} \in \hat{N}}$ is the general solution of the system of homogeneous equations

$$
0=\sum_{\hat{j} \in \hat{N}} \iint_{\hat{i}}^{(0)} f_{\hat{j}}^{(0)}\left(\phi_{\hat{i}}^{(r)}+\phi_{\hat{j}}^{(r)}-\phi_{\hat{i}}^{(r)^{\prime}}-\phi_{\hat{j}}^{(r)^{\prime}}\right) k_{\hat{i}} d \mathbf{k} d \mathbf{c}_{\hat{j}} \quad(\hat{i} \in \hat{N}) .
$$

Multiplying Equations (50) by $\phi_{\hat{i}}^{(r)}$, integrating over all values of $\mathbf{c}_{\hat{i}}$, summing over $\hat{i}$ and transforming integrals, we obtain

$$
\frac{1}{4} \sum_{\hat{i}, \hat{j} \in \hat{N}} \iiint f_{\hat{i}}^{(0)} f_{\hat{j}}^{(0)}\left(\phi_{\hat{i}}^{(r)}+\phi_{\hat{j}}^{(r)}-\phi_{\hat{i}}^{(r)^{\prime}}-\phi_{\hat{j}}^{(r)^{\prime}}\right)^{2} k_{\hat{i} j} d \mathbf{k} d \mathbf{c}_{\hat{i}} d \mathbf{c}_{\hat{j}}=0 .
$$

From (51) we conclude, that $\phi_{i}^{(r)}$ are linear combinations of the summational invariants of the collision $\psi_{i}^{(l)}$ $(l=1,2,3)$ :

$$
\phi_{\hat{i}}^{(r)}=\alpha_{\hat{i}}^{(1, r)}+\mathbf{a}^{(2, r)} \cdot m_{\hat{i}} \mathbf{c}_{\hat{i}}+\alpha^{(3, r)} \frac{1}{2} m_{\hat{i}} c_{\hat{i}}^{2},
$$

where $\alpha_{\hat{i}}^{(1, r)}$ and $\alpha^{(3, r)}$ are some, independent of $\mathbf{c}_{\hat{i}}$, scalar functions of spatial coordinates, defined by the radius vector $\mathbf{r}$, and time $t$, and $\mathbf{a}^{(2, r)}$ is a vector function of $\mathbf{r}$ and $t$ (as well as above, arbitrary functions $\mathbf{a}^{(2, r)}$ and $\alpha^{(3, r)}$ are identical for all inner components of the mixture), and, hence,

$$
\xi_{\hat{i}}^{(r)}=f_{\hat{i}}^{(0)}\left(\alpha_{\hat{i}}^{(1, r)}+\mathbf{a}^{(2, r)} \cdot m_{\hat{i}} \mathbf{c}_{\hat{i}}+\alpha^{(3, r)} \frac{1}{2} m_{\hat{i}} c_{\hat{i}}^{2}\right) \quad(\hat{i} \in \hat{N}) .
$$

To simplify further evaluations according to the expression for $f_{\hat{i}}^{(0)}$, see (18) and (31)-(33), let us rewrite (53) as

$$
\xi_{\hat{i}}^{(r)}=f_{\hat{i}}^{(0)}\left[\beta_{\hat{i}}^{(1, r)}+\mathbf{b}^{(2, r)} \cdot m_{\hat{i}}\left(\mathbf{c}_{\hat{i}}-\mathbf{u}^{(0)}\right)+\beta^{(3, r)} \frac{1}{2} m_{\hat{i}}\left(\mathbf{c}_{\hat{i}}-\mathbf{u}^{(0)}\right)^{2}\right] \quad(\hat{i} \in \hat{N}),
$$

where $\beta_{\hat{i}}^{(1, r)}, \mathbf{b}^{(2, r)}$ and $\beta^{(3, r)}$ are new functions of $\mathbf{r}$ and $t$. Family of functions $\left\{\chi_{\hat{i}}^{(r)}\right\}_{\hat{i} \in \hat{N}}$ is a solution of the system of inhomogeneous equations

$$
F_{\hat{i}}^{(r)}=\sum_{\hat{j} \in \hat{N}} \iint_{\hat{i}}^{(0)} f_{\hat{j}}^{(0)}\left(\chi_{\hat{i}}^{(r)}+\chi_{\hat{j}}^{(r)}-\chi_{\hat{i}}^{(r)^{\prime}}-\chi_{\hat{j}}^{(r)^{\prime}}\right) k_{\hat{i} j} d \mathbf{k} d \mathbf{c}_{\hat{j}} \quad(\hat{i} \in \hat{N}),
$$

where $F_{\hat{i}}^{(r)}$ denote left-hand sides of the Equations (49), taken with opposite sign.

Multiplying Equations (55) by $\psi_{\hat{i}}^{(l)} \quad(l=1,2,3)$, integrating over all values of $\mathbf{c}_{\hat{i}}$ and transforming integrals as above, we obtain as necessary condition for the existence of solutions of the system of integral Equations (55), the necessity of the fulfillment of equalities:

$$
\begin{array}{rlrl}
\int \psi_{\hat{i}}^{(1)} F_{\hat{i}}^{(r)} d \mathbf{c}_{\hat{i}} & =0 & & (\hat{i} \in \hat{N}), \\
\sum_{\hat{i} \in \hat{N}} \int \psi_{\hat{i}}^{(l)} F_{\hat{i}}^{(r)} d \mathbf{c}_{\hat{i}}=0 & & (l=2,3) .
\end{array}
$$

Among (infinitesimal) set of particular solutions of the system of Equations (55), different from each other on some solution of the system of homogeneous Equations (50), unique solution $\left\{\Phi_{\hat{i}}^{(r)}\right\}_{\hat{i} \in \hat{N}}$ may be chosen such that

$$
\begin{array}{cc}
\int \psi_{\hat{i}}^{(1)} f_{\hat{i}}^{(0)} \Phi_{\hat{i}}^{(r)} d \mathbf{c}_{\hat{i}}=0 & (\hat{i} \in \hat{N}), \\
\sum_{\hat{i} \in \hat{N}} \int \psi_{\hat{i}}^{(l)} f_{\hat{i}}^{(0)} \Phi_{\hat{i}}^{(r)} d \mathbf{c}_{\hat{i}}=0 & (l=2,3) .
\end{array}
$$


Having substituted expression for $f_{\hat{i}}^{(r)}(\hat{i} \in \hat{N})$

$$
\begin{aligned}
& f_{\hat{i}}^{(r)}=\Xi_{\hat{i}}^{(r)}+\xi_{\hat{i}}^{(r)} \\
& =f_{\hat{i}}^{(0)} \Phi_{\hat{i}}^{(r)}+f_{\hat{i}}^{(0)}\left[\beta_{\hat{i}}^{(1, r)}+\mathbf{b}^{(2, r)} \cdot m_{\hat{i}}\left(\mathbf{c}_{\hat{i}}-\mathbf{u}^{(0)}\right)+\beta^{(3, r)} \frac{1}{2} m_{\hat{i}}\left(\mathbf{c}_{\hat{i}}-\mathbf{u}^{(0)}\right)^{2}\right]
\end{aligned}
$$

in (26)-(28), taking (18), (29)-(33) and (58)-(59) into account, we obtain a system of $\operatorname{Card}(\hat{N})+4$ algebraic equations [constraint equations for asymptotic expansions (10) and (23)-(25)]:

$$
\begin{gathered}
n_{\hat{i}}^{(0)} \beta_{\hat{i}}^{(1, r)}+\frac{3}{2} n_{\hat{i}}^{(0)} k T^{(0)} \beta^{(3, r)}=n_{\hat{i}}^{(r)} \quad(\hat{i} \in \hat{N}), \\
\mathbf{u}^{(0)} \sum_{\hat{i} \in \hat{N}} m_{\hat{i}} n_{\hat{i}}^{(0)} \beta_{\hat{i}}^{(1, r)}+\hat{\rho}^{(0)} k T^{(0)} \mathbf{b}^{(2, r)}+\frac{3}{2} \hat{\rho}^{(0)} k T^{(0)} \mathbf{u}^{(0)} \beta^{(3, r)}=\sum_{s=0}^{r} \hat{\rho}^{(r-s)} \mathbf{u}^{(s)}, \\
\frac{1}{2} \sum_{\hat{i} \in \hat{N}} n_{\hat{i}}^{(0)}\left[3 k T^{(0)}+m_{\hat{i}}\left(u^{(0)}\right)^{2}\right] \beta_{\hat{i}}^{(1, r)}+\hat{\rho}^{(0)} k T^{(0)} \mathbf{u}^{(0)} \cdot \mathbf{b}^{(2, r)} \\
+\frac{3}{4} k T^{(0)}\left[5 \hat{n}^{(0)} k T^{(0)}+\hat{\rho}^{(0)}\left(u^{(0)}\right)^{2}\right] \beta^{(3, r)} \\
=\frac{3}{2} k \sum_{s=0}^{r} \hat{n}^{(r-s)} T^{(s)}+\frac{1}{2} \sum_{s=0}^{r} \sum_{q=0}^{s} \hat{\rho}^{(r-s)} \mathbf{u}^{(s-q)} \cdot \mathbf{u}^{(q)},
\end{gathered}
$$

from which we find expressions for functions $\beta_{\hat{i}}^{(1, r)}(\mathbf{r}, t), \mathbf{b}^{(2, r)}(\mathbf{r}, t)$ and $\beta^{(3, r)}(\mathbf{r}, t)$ through (variable) coefficients of asymptotic expansions of the particle number density of $\hat{i}$-component, of the mean mass velocity and of the temperature of inner components of the mixture

$$
\begin{aligned}
& \beta_{\hat{i}}^{(1, r)}= \frac{n_{i}^{(r)}}{n_{\hat{i}}^{(0)}}-\frac{3}{2} \frac{1}{\hat{n}^{(0)} T^{(0)}}\left[\sum_{s=0}^{r}\left(\hat{n}^{(r-s)} T^{(s)}\right)-\hat{n}^{(r)} T^{(0)}\right] \\
&-\frac{1}{2} \frac{1}{\hat{n}^{(0)} k T^{(0)}}\left[\sum_{s=0}^{r} \sum_{q=0}^{s} \hat{\rho}^{(r-s)} \mathbf{u}^{(s-q)} \cdot \mathbf{u}^{(q)}-\hat{\rho}^{(r)}\left(u^{(0)}\right)^{2}\right] \\
&+\frac{1}{\hat{n}^{(0)} k T^{(0)}} \mathbf{u}^{(0)} \cdot\left[\sum_{s=0}^{r}\left(\hat{\rho}^{(r-s)} \mathbf{u}^{(s)}\right)-\hat{\rho}^{(r)} \mathbf{u}^{(0)}\right], \\
& \beta^{(3, r)}= \frac{k}{\hat{n}^{(0)}\left(k T^{(0)}\right)^{2}}\left[\sum_{s=0}^{r}\left(\hat{n}^{(r-s)} T^{(s)}\right)-\hat{n}^{(r)} T^{(0)}\right] \\
&+\frac{1}{3} \frac{1}{\hat{n}^{(0)}\left(k T^{(0)}\right)^{2}}\left[\sum_{s=0}^{r} \sum_{q=0}^{s} \hat{\rho}^{(r-s)} \mathbf{u}^{(s-q)} \cdot \mathbf{u}^{(q)}-\hat{\rho}^{(r)}\left(u^{(0)}\right)^{2}\right] \\
&-\frac{2}{3} \frac{1}{\hat{n}^{(0)}\left(k T^{(0)}\right)^{2}} \mathbf{u}^{(0)} \cdot\left[\sum_{s=0}^{r}\left(\hat{\rho}^{(r-s)} \mathbf{u}^{(s)}\right)-\hat{\rho}^{(r)} \mathbf{u}^{(0)}\right] .
\end{aligned}
$$

Then the fulfillment of equalities (56)-(57) can be considered as the differential equations, the $r$-order equations of gas dynamics, for finding $n_{\hat{i}}^{(r-1)}, \mathbf{u}_{\hat{i}}^{(r-1)}, T_{\hat{i}}^{(r-1)}(r=1,2, \ldots)$.

The partial solution of the system of inhomogeneous Equations (55) $\left\{\Phi_{\hat{i}}^{(r)}\right\}_{\hat{i} \in \hat{N}}$, satisfying (58)-(59), may be constructed, for example, using expansion of $\Phi_{\hat{i}}^{(r)}\left(\mathbf{c}_{\hat{i}}\right)$ in series in terms of Sonine polynomials with expansion 
coefficients, depending on $\mathbf{r}$ and $t$ (see [2] or [4]); such construction proves existence of solutions of the system of integral Equations (49).

For $r \geq 1$ the velocity distribution functions of outer components of gas mixture $f_{i}^{(r)}$ may be similarly found from the integral equations system (16):

$$
f_{\bar{i}}^{(r)}=\Xi_{\bar{i}}^{(r)}+\xi_{\bar{i}}^{(r)}=f_{\bar{i}}^{(0)} \Phi_{\bar{i}}^{(r)}+f_{\bar{i}}^{(0)}\left[\beta_{\bar{i}}^{(1, r)}+\mathbf{b}_{\bar{i}}^{(2, r)} \cdot m_{\bar{i}}\left(\mathbf{c}_{\bar{i}}-\mathbf{u}_{\bar{i}}^{(0)}\right)+\beta_{\bar{i}}^{(3, r)} \frac{1}{2} m_{\bar{i}}\left(\mathbf{c}_{\bar{i}}-\mathbf{u}_{\bar{i}}^{(0)}\right)^{2}\right]
$$

where

$$
\begin{aligned}
& \beta_{\bar{i}}^{(1, r)}=\frac{n_{i}^{(r)}}{n_{\bar{i}}^{(0)}}-\frac{3}{2} \frac{1}{n_{i}^{(0)} T_{\bar{i}}^{(0)}}\left[\sum_{s=0}^{r}\left(n_{\bar{i}}^{(r-s)} T_{\bar{i}}^{(s)}\right)-n_{i}^{(r)} T_{i}^{(0)}\right] \\
& -\frac{1}{2} \frac{1}{n_{i}^{(0)} k T_{i}^{(0)}}\left[\sum_{s=0}^{r} \sum_{q=0}^{s} \rho_{i}^{(r-s)} \mathbf{u}_{i}^{(s-q)} \cdot \mathbf{u}_{i}^{(q)}-\rho_{i}^{(r)}\left(u_{i}^{(0)}\right)^{2}\right] \\
& +\frac{1}{n_{i}^{(0)} k T_{i}^{(0)}} \mathbf{u}_{i}^{(0)} \cdot\left[\sum_{s=0}^{r}\left(\rho_{\bar{i}}^{(r-s)} \mathbf{u}_{i}^{(s)}\right)-\rho_{i}^{(r)} \mathbf{u}_{i}^{(0)}\right], \\
& \mathbf{b}_{\bar{i}}^{(2, r)}=\frac{1}{\rho_{\bar{i}}^{(0)} k T_{i}^{(0)}}\left[\sum_{s=0}^{r}\left(\rho_{\bar{i}}^{(r-s)} \mathbf{u}_{i}^{(s)}\right)-\rho_{\bar{i}}^{(r)} \mathbf{u}_{\bar{i}}^{(0)}\right], \\
& \beta_{\bar{i}}^{(3, r)}=\frac{k}{n_{\bar{i}}^{(0)}\left(k T_{\bar{i}}^{(0)}\right)^{2}}\left[\sum_{s=0}^{r}\left(n_{\bar{i}}^{(r-s)} T_{\bar{i}}^{(s)}\right)-n_{\bar{i}}^{(r)} T_{\bar{i}}^{(0)}\right] \\
& +\frac{1}{3} \frac{1}{n_{i}^{(0)}\left(k T_{i}^{(0)}\right)^{2}}\left[\sum_{s=0}^{r} \sum_{q=0}^{s} \rho_{i}^{(r-s)} \mathbf{u}_{i}^{(s-q)} \cdot \mathbf{u}_{i}^{(q)}-\rho_{i}^{(r)}\left(u_{i}^{(0)}\right)^{2}\right] \\
& -\frac{2}{3} \frac{1}{n_{i}^{(0)}\left(k T_{i}^{(0)}\right)^{2}} \mathbf{u}_{i}^{(0)} \cdot\left[\sum_{s=0}^{r}\left(\rho_{\bar{i}}^{(r-s)} \mathbf{u}_{i}^{(s)}\right)-\rho_{\bar{i}}^{(r)} \mathbf{u}_{i}^{(0)}\right] .
\end{aligned}
$$

The fulfillment of analogous (56)-(57) equalities

$$
\int \psi_{\breve{i}}^{(l)} F_{\breve{i}}^{(r)} d \mathbf{c}_{\breve{i}}=0 \quad(\breve{i} \in \breve{N}, l=1,2,3)
$$

can be considered as the differential equations, the $r$-order equations of gas dynamics, for finding $n_{i}^{(r-1)}, \mathbf{u}_{i}^{(r-1)}$, $T_{\bar{i}}^{(r-1)}(r=1,2, \ldots)$.

\section{The System of First Order Equations of Multicomponent Non-Equilibrium Gas Dynamics}

Let us consider in more detail the system of infinitesimal first order Equations (56)-(57), (71) $(r=1)$, derived above as the necessary (and sufficient) condition of the solution existence of the first order integral equations system (14), (16) $(r=1)$.

To simplify transformations, according to the expressions for velocity distribution functions of particles of infinitesimal zero order (18), (35), functions $\Psi_{\hat{i}}^{(l)}, \Psi_{i}^{(l)}$ may be used in (56)-(57), (71) $(r=1)$ rather than functions $\psi_{\hat{i}}^{(l)}, \psi_{\dot{i}}^{(l)}$, respectively:

$$
\begin{gathered}
\Psi_{i}^{(1)}=m_{i}, \\
\Psi_{i}^{(2)}=m_{i} \mathbf{C}_{i}, \\
\Psi_{i}^{(3)}=\frac{1}{2} m_{i} C_{i}^{2},
\end{gathered}
$$


for inner components $\mathbf{C}_{\hat{i}}=\mathbf{c}_{\hat{i}}-\mathbf{u}^{(0)}$, for outer components $\mathbf{C}_{\tilde{i}}=\mathbf{c}_{\tilde{i}}-\mathbf{u}_{\hat{i}}^{(0)}$.

At transformation of differential parts of the Equations (56)-(57) and (71) we use equalities:

$$
\begin{gathered}
\int \Psi_{i}^{(l)} \frac{\partial f_{i}^{(0)}}{\partial t} d \mathbf{c}_{i}=\frac{\partial}{\partial t} \int \Psi_{i}^{(l)} f_{i}^{(0)} d \mathbf{c}_{i}-\int \frac{\partial \Psi_{i}^{(l)}}{\partial t} f_{i}^{(0)} d \mathbf{c}_{i}=\frac{\partial\left(n_{i}{\overline{\Psi_{i}^{(l)}}}^{(0)}\right)}{\partial t}-n_{i} \frac{{\overline{\Psi_{i}^{(l)}}}^{(0)}}{\partial t}, \\
\int \Psi_{i}^{(l)} \mathbf{c}_{i} \cdot \frac{\partial f_{i}^{(0)}}{\partial \mathbf{r}} d \mathbf{c}_{i}=\frac{\partial}{\partial \mathbf{r}} \cdot \int \Psi_{i}^{(l)} \mathbf{c}_{i} f_{i}^{(0)} d \mathbf{c}_{i}-\int \mathbf{c}_{i} \cdot \frac{\partial \Psi_{i}^{(l)}}{\partial \mathbf{r}} f_{i}^{(0)} d \mathbf{c}_{i}=\frac{\partial}{\partial \mathbf{r}} \cdot n_{i}{\overline{\Psi_{i}^{(l)} \mathbf{c}_{i}}}^{(0)}-n_{i} \mathbf{c}_{i} \cdot \frac{\partial \Psi_{i}^{(l)}}{\partial \mathbf{r}} \\
\int \Psi_{i}^{(l)} \frac{\mathbf{X}_{i}}{m_{i}} \cdot \frac{\partial f_{i}^{(0)}}{\partial \mathbf{c}_{i}} d \mathbf{c}_{i}=-\int\left(\frac{\partial}{\partial \mathbf{c}_{i}} \cdot \Psi_{i}^{(l)} \frac{\mathbf{X}_{i}}{m_{i}}\right) f_{i}^{(0)} d \mathbf{c}_{i}=-n_{i} \frac{\partial}{\partial \mathbf{c}_{i}} \cdot \Psi_{i}^{(l)}{\frac{\mathbf{X}_{i}}{m_{i}}}^{(0)}
\end{gathered}
$$

In (75)-(77) the bar above symbol with index ${ }^{(0)}$ denotes the average of the value:

$$
\bar{V}^{(0)}=\frac{1}{n_{i}} \int V f_{i}^{(0)} d \mathbf{c}_{i} ;
$$

$\mathbf{r}$ and $\mathbf{c}_{i}$ are considered as independent variables. At averaging in (77) it is assumed, that external force $\mathbf{X}_{i}$, acting on the particle of species $i$, is independent of the particle velocity, it is assumed also, that integrals, depending on external forces $\mathbf{X}_{i}$, are convergent, and product $\Psi_{i}^{(l)} \mathbf{X}_{i} f_{i}^{(0)}$ tends to zero, when $\mathbf{c}_{i}$ tends to infinity.

After simple transformations from (56)-(57) and (71) $(r=1)$ we obtain following system of infinitesimal first order equations of multicomponent non-equilibrium gas dynamics:

$$
\begin{aligned}
& \frac{\partial n_{\hat{i}}^{(0)}}{\partial t}=-\frac{\partial}{\partial \mathbf{r}} \cdot n_{\hat{i}}^{(0)} \mathbf{u}^{(0)} \quad(\hat{i} \in \hat{N}), \\
& \hat{\rho}^{(0)} \frac{\partial \mathbf{u}^{(0)}}{\partial t}+\frac{\partial}{\partial \mathbf{r}} \cdot \hat{\mathrm{p}}^{(0)}+\sum_{\hat{i} \in \hat{N}, \hat{j} \in \bar{N}} \mathbf{J}_{p, \hat{j}}^{(0)}=\sum_{i \in \hat{N}} n_{\hat{i}}^{(0)} \mathbf{X}_{\hat{i}}-\hat{\rho}^{(0)} \mathbf{u}^{(0)} \cdot \frac{\partial}{\partial \mathbf{r}} \mathbf{u}^{(0)}, \\
& \frac{\partial \hat{E}^{(0)}}{\partial t}+\frac{\partial}{\partial \mathbf{r}} \cdot \hat{\mathbf{q}}^{(0)}+\hat{\mathrm{p}}^{(0)}: \frac{\partial \mathbf{u}^{(0)}}{\partial \mathbf{r}}+\sum_{\hat{i} \in \hat{N}, \hat{j} \in \tilde{N}} J_{E, \hat{j} j}^{(0)}=-\frac{\partial}{\partial \mathbf{r}} \cdot \hat{E}^{(0)} \mathbf{u}^{(0)}, \\
& \frac{\partial n_{i}^{(0)}}{\partial t}=-\frac{\partial}{\partial \mathbf{r}} \cdot n_{\bar{i}}^{(0)} \mathbf{u}_{\bar{i}}^{(0)} \quad(\breve{i} \in \breve{N}), \\
& n_{i}^{(0)} m_{\bar{i}} \frac{\partial \mathbf{u}_{i}^{(0)}}{\partial t}+\frac{\partial}{\partial \mathbf{r}} \cdot p_{\bar{i}}^{(0)}+\sum_{j \neq i} \mathbf{J}_{p, i \bar{j}}^{(0)}=n_{i}^{(0)} \mathbf{X}_{\bar{i}}-n_{\bar{i}}^{(0)} m_{\bar{i}} \mathbf{u}_{\bar{i}}^{(0)} \cdot \frac{\partial}{\partial \mathbf{r}} \mathbf{u}_{i}^{(0)} \quad(\breve{i} \in \breve{N}), \\
& \frac{\partial E_{\bar{i}}^{(0)}}{\partial t}+\frac{\partial}{\partial \mathbf{r}} \cdot \mathbf{q}_{\bar{i}}^{(0)}+\mathrm{p}_{\bar{i}}^{(0)}: \frac{\partial \mathbf{u}_{i}^{(0)}}{\partial \mathbf{r}}+\sum_{j \neq \bar{i}} J_{E, \grave{j}}^{(0)}=-\frac{\partial}{\partial \mathbf{r}} \cdot E_{\bar{i}}^{(0)} \mathbf{u}_{\bar{i}}^{(0)} \quad(\breve{i} \in \breve{N}) .
\end{aligned}
$$

In accordance with the general definition of pressure tensor of $i$-component of gas mixture

$$
\mathrm{p}_{i} \stackrel{\text { def }}{=} \int m_{i}\left(\mathbf{c}_{i}-\mathbf{u}_{i}\right)\left(\mathbf{c}_{i}-\mathbf{u}_{i}\right) f_{i} d \mathbf{c}_{i}
$$

and with the general definition of $i$-component heat flux vector

$$
\mathbf{q}_{i} \stackrel{\text { def }}{=} \int \frac{1}{2} m_{i}\left(\mathbf{c}_{i}-\mathbf{u}_{i}\right)^{2}\left(\mathbf{c}_{i}-\mathbf{u}_{i}\right) f_{i} d \mathbf{c}_{i}
$$

(cf. with [2], Chapter 2, §§ 3, 4) in (79)-(84)

$$
\left.\hat{\mathrm{p}}^{(0)}=\sum_{\hat{i} \in \hat{N}} n_{\hat{i}}^{(0)} m_{\hat{i}} \overline{\left(\mathbf{c}_{\hat{i}}-\mathbf{u}^{(0)}\right)\left(\mathbf{c}_{\hat{i}}-\mathbf{u}^{(0)}\right.}\right)^{(0)}=\hat{n}^{(0)} k T^{(0)} \mathrm{U}=\hat{p}^{(0)} \mathrm{U}
$$


is inner components pressure tensor of zero order, $\hat{p}^{(0)}$ is inner components hydrostatic pressure of zero order, $\mathrm{U}$ is the unit tensor, double product of two second rank tensors $\mathrm{w}$ and $\mathrm{w}^{\prime}$ ([2], Chapter 1, § 3 ) is the scalar $\mathrm{w}: \mathrm{w}^{\prime}=\sum_{\alpha} \sum_{\beta} w_{\alpha \beta} \mathrm{w}_{\beta \alpha}^{\prime}=\mathrm{w}^{\prime}: \mathrm{w}$,

$$
\hat{\mathbf{q}}^{(0)}=\frac{1}{2} \sum_{\hat{i} \in \hat{N}} n_{\hat{i}}^{(0)} m_{\hat{i}} \overline{\left(\mathbf{c}_{\hat{i}}-\mathbf{u}^{(0)}\right)^{2}\left(\mathbf{c}_{\hat{i}}-\mathbf{u}^{(0)}\right)}{ }^{(0)}=0
$$

is inner components heat flux vector of zero order,

$$
\hat{E}^{(0)}=\frac{1}{2} \sum_{\hat{i} \in \hat{N}} n_{\hat{i}}^{(0)} m_{\hat{i}} \overline{\left(\mathbf{c}_{\hat{i}}-\mathbf{u}^{(0)}\right)^{2}}(0)=\frac{3}{2} \hat{n}^{(0)} k T^{(0)}
$$

is zero order internal energy of particles of inner components per unit volume, which is equal, in this case, to energy of their translational chaotic motion, however, the energy transfer equations, written in form (81) and (84) can be used in more general cases as well (cf. with [4], Chapter 7, § 6), in (87)-(89) averaging (78) is performed with Maxwell function $f_{\hat{i}}^{(0)}$ from (18);

$$
\mathrm{p}_{\bar{i}}^{(0)}=n_{\bar{i}}^{(0)} m_{\bar{i}} \overline{\left(\mathbf{c}_{\bar{i}}-\mathbf{u}_{\bar{i}}^{(0)}\right)\left(\mathbf{c}_{\bar{i}}-\mathbf{u}_{\bar{i}}^{(0)}\right)^{(0)}}=n_{\bar{i}}^{(0)} k T_{\bar{i}}^{(0)} \mathrm{U}=p_{\bar{i}}^{(0)} \mathrm{U}
$$

is $\breve{i}$-component pressure tensor of zero order, $p_{\bar{i}}^{(0)}$ is $\breve{i}$-component hydrostatic pressure of zero order,

$$
\mathbf{q}_{\bar{i}}^{(0)}=\frac{1}{2} n_{\bar{i}}^{(0)} m_{\bar{i}} \overline{\left(\mathbf{c}_{\check{i}}-\mathbf{u}_{\bar{i}}^{(0)}\right)^{2}\left(\mathbf{c}_{\check{i}}-\mathbf{u}_{\bar{i}}^{(0)}\right)}=0
$$

is $\breve{i}$-component heat flux vector of zero order,

$$
E_{i}^{(0)}=\frac{1}{2} n_{i}^{(0)} m_{\bar{i}} \overline{\left(\mathbf{c}_{\check{i}}-\mathbf{u}_{i}^{(0)}\right)^{2}}=\frac{3}{2} n_{i}^{(0)} k T_{i}^{(0)}
$$

is zero order internal energy of particles of $\breve{i}$-component per unit volume, in (90)-(92) averaging (78) is performed with Maxwell function $f_{\bar{i}}^{(0)}$ from (35).

General analytic expressions for integrals $\mathbf{J}_{p, i j}^{(0)}, J_{E, i j}^{(0)}$ from (80), (81) and (83), (84), that depend on the interaction cross-section, can be derived in general case, when separate components (with Maxwell velocity distribution function of particles) have different mean velocities and temperatures.

System of infinitesimal first order equations of multicomponent non-equilibrium gas dynamics (79)-(84) is proposed to use for describing turbulent flows

\section{References}

[1] Hilbert, D. (1912) Grundzüge einer Allgemeinen Theorie der Linearen Integralgleichungen. Teubner, Leipzig and Berlin. (In German)

[2] Chapman, S. and Cowling, T.G. (1952) The Mathematical Theory of Non-uniform Gases. Cambridge University Press, Cambridge.

[3] Struminskii, V.V. (1974) Influence of Diffusion Velocity on Flow of Gas Mixtures. Prikladnaya Mathematica i Mechanica [Applied Mathematics and Mechanics], 38, 203-210. (In Russian)

[4] Hirschfelder, J.O., Curtiss, Ch.F. and Bird, R.B. (1954) Molecular Theory of Gases and Liquids. Wiley, New York. 


\section{Submit or recommend next manuscript to SCIRP and we will provide best service for you:}

Accepting pre-submission inquiries through Email, Facebook, LinkedIn, Twitter, etc.

A wide selection of journals (inclusive of 9 subjects, more than 200 journals)

Providing 24-hour high-quality service

User-friendly online submission system

Fair and swift peer-review system

Efficient typesetting and proofreading procedure

Display of the result of downloads and visits, as well as the number of cited articles

Maximum dissemination of your research work

Submit your manuscript at: http://papersubmission.scirp.org/ 